\title{
Confined free motion under a dipole potential
}

\author{
R. Sánchez-Martínez \\ Facultad de Física, Universidad Veracruzana, \\ Xalapa de Enríquez CP 91000 Veracruz, México. \\ e-mail:rodrigo96fml@gmail.com \\ H. N. Núñez-Yépez \\ Departamento de Física, Universidad Autónoma Metropolitana, \\ Unidad Iztapalapa, Apartado Postal 55-534, Iztapalapa CP 09340 CDMX, México. \\ e-mail: nyhn@xanum.uam.mx \\ A. L. Salas-Brito \\ Laboratorio de Sistemas Dinámicos, Departamento de Ciencias Básicas, Universidad Autónoma Metropolitana, \\ Unidad Azcapotzalco, Apartado Postal 21-267, Coyoacan CP 04000 CDMX, México. \\ e-mail: asalasbrito@yahoo.com
}

Received 5 March 2020; accepted 15 May 2020

\begin{abstract}
The classical motion of a particle in an attractive dipolar potential, $U_{\text {dip }}(q)=-k / q^{2}$, and free motion along a curve in phase space are proven to be equivalent. We also prove that the singularity at $q=0$ in the dipolar potential is strong enough as to prevent the flow of particles from one side of the singularity to the other. This effect does not depend on whether the dipole potential is regarded as attractive $(k>0)$ or as repulsive $(k<0)$. All the proofs are given using the Hamitonian formalism; thus, they may be used for illustrating the power the Hamiltonian approach confers in analysing mecanical systems. The discussion is kept within the reach of advanced undergraduate or beginning graduate students of Hamiltonian mechanics.
\end{abstract}

Keywords: Inverse square potential; equivalence to free motion; impenetrability of the origin.

PACS: 45.20.d; 45.05.+x

\section{Introduction}

There is no simpler physical system than a particle moving free from any interaction. That is, a point-mass moving in a straight line with constant velocity. If we try to imagine an interacting classical particle in canonical phase space (PS) moving under similar conditions, we would need to substitute the interaction potential for something else, which, taking a clue from gravitation [1], we may take as the PS geometry - not the geometry of the spacetime. That is, we would need a warped PS as the arena in which the motion unfolds. But how can it be that we may change the PS while mantaining the effect of the interaction on the particle motion? It is one of the aims of this paper is to try to answer this question in a way as simple as possible.

Hamiltonian mechanics [2-5] may come to our minds as the formulation of dynamics appropriate for tackling the aforementioned problem. The Hamiltonian formulation allows us to peer deeper into the fundamental structure of classical mechanics, allowing us to prove surprising general results as Poincaré's or Liouville's theorems, or even the famous KAM theorem [2,6]. And, as we are going to exhibit in this work, the features of such formulation of mechanics also allow us to pose and solve our problem. We are going to exhibit that the motion under the radial inverse square potential is equivalent to geodesic motion on a PS curve. We should notice that our result might also be demonstrated us-
DOI: https://doi.org/10.31349/RevMexFisE.17.272

ing the global approach of Moser [7] or the local approach discussed in [8-10].

The classical systems in which this feature holds, are termed geometric, that is, systems which are equivalent to a free, geodesic motion in a curved space. This is a feature first proved by Moser [7] for the Coulomb problem, though physicist had used the result several times before Moser's formal proof $[11,12]$. It is the purpose of this paper to stablish that such equivalence also holds for the $1 \mathrm{D}$ inverse square potential and to show that the singularity at $q=0$ in the dipolar potential acts as a repulsive one, resulting in the confinement of the particle to just one side of the singularity.

We first aim to prove that the motion of a particle moving in the $1 \mathrm{D}$ dipole potential, $U_{\operatorname{dip}}(q)=-k / q^{2}, k>0$ [13], and hence described by the Hamiltonian,

$$
H=\frac{p^{2}}{2 m}-\frac{k}{q^{2}},
$$

may be transformed using classical canonical (Hamiltonian) techniques into free, purely geodesic, motion in a curve in the transformed PS of the problem. However, at difference with Moser more global approach [7], we here make use of canonical transformations in the Hamiltonian formulation of classical mechanics to establish the equivalence, an approach more appropriate for the pedagogical nature of this work. Note also that Hamiltonian of (1) describes a two body problem from the viewpoint of its center of mass. Therefore, we are allowed to study the equivalent motion of a single mass-point. 


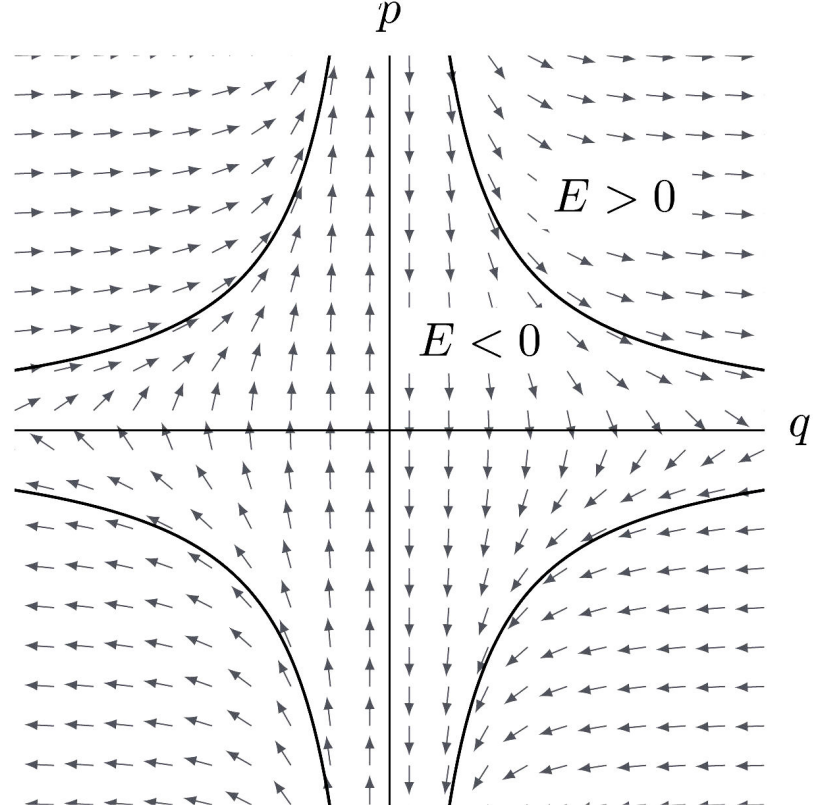

FIGURE 1. The vector field associated with Hamiltonain of (1). This plot clearly illustrates that the particle never reaches the singularity at $q=0$ and thus that it remains in just one side of the singularity without ever crossing to the other side.

\section{Impenetrability of the dipolar potential}

The Hamiltonian of (1) is time independent or autonomous; therefore, the energy $(\mathrm{E})$ is a constant of motion. Another feature of the motion in the 1D dipole potential is the complete separation between the right and left regions of the origin (the so-called space splitting), which is the site of the singularity. This can be proven as follows, consider the sign of the coordinate $q$, and let us define the variable $s$ as $s=1$ if and only if $q>0$ and $s=-1$ if and only if $q<0$; this quantity carries the information about the side of the origin in which the particle moves. We are going to show that $s$ is a constant, independent of the sign of $k$, meaning that the particle never crosses from one side of the origin to the other. To this end, it suffices to note that the following Poisson bracket vanishes

$$
\{s, H\}=0,
$$

meaning that the sign of the particle's coordinate $s$ is a constant of the motion, or that the particle never reaches nor crosses the origin ${ }^{i}$. The particle gets confined to move on a single side of the singularity. Thus we have proved the origin to be impenetrable for a particle moving in a dipole potential. This feature is illustrated by the phase plot in Fig. 1. Notice that such impenetrability does not depend on the sign of the coupling constant $k$.

\section{The particle trajectories}

What are the trajectories a particle follows in the system? To answer this, let us begin writing the energy $E$

$$
E=\frac{p^{2}}{2 m}-\frac{k}{q^{2}}
$$

That is, the particle moves following the constant energy curves in PS, which we may obtain from Eq. (3) as

$$
p(q)= \pm \sqrt{2 m E+\frac{2 m k}{q^{2}}},
$$

describing the trajectories of particles moving in the dipolar system for any value of the energy. Notice that, despite attracting particles more strongly than the Coulomb potential, this potential is actually weaker at large distances from the center of force enough to prevent the bounding of particlesexcept, as we show below, in the case of vanishing energy. Equation (4) describes the unbound trajectories of particles moving under a dipole potential.

If the energy vanishes $(E=0)$, the curves are hyperbolas as, in such a case,

$$
p= \pm \frac{\sqrt{2 m k}}{q} .
$$

Such curves divides the phase space in two a zone of trajectories with positive energy and another with trajectories of negative energy. You should also be aware that if $E=0$, the particle falls to the center, where it bounces off to infinity, then falling down and bouncing off again in an endless cycle. A particle moving in an inverse square potential at negative energies is never trapped. The only bounded orbit, is the 'falling and bouncing' motion at vanishing energy.

\section{Equivalence to free motion on a curve in phase space}

To prove the mentioned equivalence, we first need to notice that we may express the Hamiltonian Eq. (1) as

$$
H=\frac{p^{2}}{2 m}\left(1-\frac{2 m k}{p^{2} q^{2}}\right)
$$

and, as suggested by the previous expression and using the freedom afforded by the Hamiltonian approach, we may define a new canonical momentum $P$ as

$$
P=p \sqrt{1-\frac{2 m k}{p^{2} q^{2}}}
$$

From Eq. (7), and after tinkering a bit, we may find the generating function $G(P, q)$, and the conjugate variable $Q$, making the variables $Q$ and $P$ canonical, as follows. If we set

$$
p=\frac{\partial G}{d q},
$$

then, a simple integration gives the generating function

$$
\begin{aligned}
G(P, q) & =\sqrt{P^{2} q^{2}+2 m k}+2 m k \\
& \times\left[\ln (P q)-\ln \left(\sqrt{2 m k}+\sqrt{P^{2} q^{2}+2 m k}\right)\right] .
\end{aligned}
$$



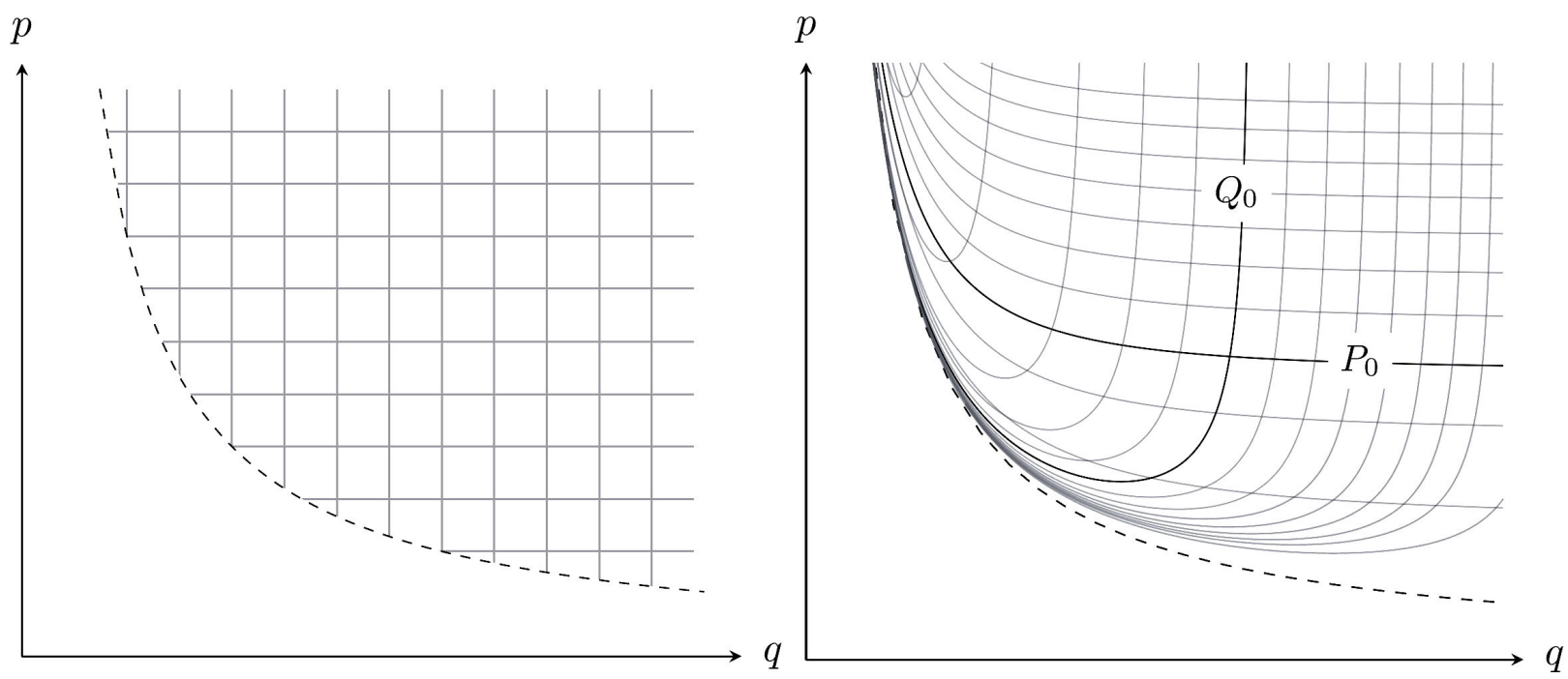

FIGURE 2. On the left, representation of the straight lines, $p$ constant and $q$ constant, in the original phase space. On the right, representation of the corresponding curves in the transformed PS of the system. We have hightlighted two geodesics, $Q=Q_{0}$ and $P=P_{0}$.

As we have said, the above expression, Eq. (7), is going to help us to transform the Hamiltonian Eq. (1) into the free particle form $P^{2} / 2 m$. Of course, we would still need a new cordinate, $Q$, defined for making sure the whole transformation is canonical. Such canonical variable can also be found using $G(P, q)$ above, as

$$
Q=\frac{G(P, q)}{\partial P}
$$

Therefore, the new PS canonical coordinate $Q$ has to be

$$
Q=\frac{q}{\sqrt{1-\frac{2 m k}{p^{2} q^{2}}}} .
$$

In the new canonical coordinates $(Q, P)$, the new Hamiltonian of the system has only a kinetic energy term,

$$
\tilde{H}(P, Q)=\frac{P^{2}}{2 m},
$$

hence the new momentum $P$ is a constant in time. The Hamiltonian of a particle moving in a dipole potential has been transformed into a free particle form.

It is important to emphasize that the free motion occurs not in the original PS, but in a warped one in which the particle's trajectory is just a geodesic. As it should be clear by the previous discussion, such warping is the cost for the suppression of the explicit appearance of the interaction potential. This could be visualized in the Fig. 2 below.

We may additionally describe the trajectories in the original PS starting from the ones in the transformed PS. In this transformed PS, we have

$$
\begin{aligned}
& P=P_{0} \quad \text { a constant }, \\
& Q=P_{0} t+Q_{0} .
\end{aligned}
$$

And, if we use the canonical transformations from Eqs. (7) and (11), we may get the solution to the problem expressed in the original coordinates. We leave this task as an exercise for the readers. Another point worth dicussing is the possibility of doing this transformation for other potentials. The answer to the reviewer's question we think should be in the affirmative as you may conclude by yourself just by following our dicussion and taking a look to the Refs. [8,9].

\section{Conclusions}

In this paper, we have intended to illustrate the flexibility afforded to us by the mehods of Hamiltonian mechanics for analysing the motion of a particle under the inverse square potential $U_{\operatorname{dip}}(q)=-k / q^{2}$ with a positive coupling constant $k$. It is to be noted that this potential is both stronger than Coulomb's near the origin, and weaker as $|q| \rightarrow \infty$. We have also shown that, despite the stronger attraction than in the Coulomb case, the inverse square potential behaves at the origin as an impenetrable barrier exhibitting what it is known as the "space splitting" phenomena [14]. This happens because the sign of the coordinate $s$ is a constant of the motion. This behavior may be extended to the quantum case, where it shows itself as a superselection rule [18].

We must recognize that the $-k / q^{2}$ potential is rather problematic because, being an attractive potential, it may be regarded as an impenetrable barrier - for an early discussion of such kind of trouble see [15]. On the other hand, such potential may be regarded as the radial potential associated with the interaction of moving electrons within the electric field of a stationary polar molecule, as the actual field is the dipolar one $-p \cos \theta / q^{2}$, were $q$ and $\theta$ are polar coordinates in a plane, $p$ is the dipole moment. Therefore, Hamiltonian Eq. (1) describes the motion of electrons coming towards the singularity directly from the direction $\theta=0$. In fact, this 
classical study may help to clarify some features of the scattering cross section of electrons on molecules with a permanent electric dipole moment [16]. We must pinpoint also that by applying the inverse canonical transformation to the solution described here, we may uncover the precise solution of the problem in the original PS. Such explicit calculation can be made, but it requires too much space, it would be better to have a look to a simpler example in which the suggested procedure was explicitly applied. Such example is the harmonic oscillator, whose discussion using a technique similar to one used here, was published recently with all details in [10].

We have shown that, in spite of it being an interaction stronger than Coulomb's, the Hamiltonian of the problem, that of Eq. (1), may be regarded as analogous to that of a particle moving freely on a curve in phase space, as it is shown in Fig. 2. You should notice that this curve is traversed from an extreme to the other in cyclic fashion and that the effect of the singularity at $q=0$ gets transformed into the two motions exhibitted in the transformed coordinates. This is analogous to the case of the two circular motions into which the effect of the bouncing occurring in the classical one-dimensional hydrogen atom is canonically transformed - as described in [12]. Furthermore, the particle under the dipolar potential avoids approaching the origin, as the vector field of the system clearly exhibits, see Fig. 1. If you aim directly towards the singularity, on getting there the particle bounces back to the initial position and, after coming to a rest momentarily, it gets attracted again towards the origin. Such bouncing and returning motion happens indefinitely. In a sense, we have an example of a classical system which is both free and confined by a singularity -in the ranges $(0, \infty)$ or $(-\infty, 0)$ - but unable to move through the origin despite its free-like behavior. The bouncing at the origin repeats itself an infinite number of times.

In summary, we think that a classical discussion of this problem may teach a lot about what we may accomplish by the purely classical analysis of potential problems notwithstanding the fact the final aim were to analyse the problem in a quantum setting. The problem analized in this paper may be regarded as the classical 1D version of the interaction of an electron with a polar molecule, as the discussion in [16] may show.

Finally, we mention that this paper allows one of us (ALSB) to write a correction to another work whose conclussions are correct, but whose analysis is badly written and wrongly explained [17]. We should emphasize that the responsability for the errors of that paper is exclusively ALSB's, none other of the participants in that work has any responsability for any such mistakes. $i$. To establish Eq. (2) we use $\partial s / \partial x=0$, not $\partial s / \partial x=\delta(x)$. This is so because we are working in a purely classical setting. In such a realm, it is difficult, if not altogether impossible, to give meaning to a distribution like Dirac's — which, on the other hand, it is easily understood as an operator in quantum mechanics.

1. C. W. Misner, K. S. Thorne, and J. A. Wheeler, Gravitation (W. H. Freeman, San Francisco, 1977).

2. L. Landau and E. M. Lifshitz, Mechanics (Pergamon Press, Oxford, 1978).

3. V. I. Arnold, Mathematical Methods of Classical Mechanics (Springer-Verlag, New York, 1978), https://doi.org/ 10.1007/978-1-4757-1693-1

4. H. Goldstein, C. Poole, and J. Safko, Classical Mechanics, 3rd ed. (Addison-Wesley, San Francisco, 2002).

5. R. Talman, Geometric Mechanics, 1st ed. (Wiley, New York, 2000).

6. D. Treschev and O. Zubelevich, Introduction to the Perturbation Theory of Hamiltonian Systems (Springer-Verlag, Berlin, 2009), pp. 23-58, https://doi.org/10.1007/ 978-3-642-03028-4_2

7. J. Moser, Regularization of Kepler's Problem and the Averaging Method on a Manifold, Comm. Pure Appl. Math. 23 (1970) 609, https://doi.org/10.1002/cpa.3160230406.

8. E. N. Glass and J. J. G. Scanio, Canonical transformation to the free particle, J. J. Scanio, Am. J. Phys. 45 (1977) 344, https://doi.org/10.1119/1.10618

9. A. Gómez-Trapote, Rev. Mex. Fis 48 (2002) 4.
10. R. Sánchez-Martínez, H. N. Núñez-Yépez, A. L. Salas-Brito, Rev. Mex. Fis. E 17 (2020) 6,

11. R. J. Finkelstein, Symmetry Group of the Hydrogen Atom, J. Math. Phys. 8 (1967) 443, https : / doi.org/10.1063/ 1.1705211 .

12. L. J. Boya, M. M. Kmiecik, and A. Bohm, Hydrogen atom in one dimension, Phys. Rev. A 37 (1988) 3567, https: //doi.org/10.1103/PhysRevA.37.3567

13. J. M. Lévy-Leblond, Nonrelativistic particles and wave equations, Comm. Math. Phys. 6 (1967) 286, https://doi. org/10.1007/BF01646020

14. U. Oseguera and M. de Llano, Two singular potentials: The space-splitting effect, J. Math. Phys. 34 (1993) 4575, https : //doi.org/10.1063/1.530358

15. C. Zhu and J. R. Klauder, Classical symptoms of quantum illnesses, Am. J. Phys. 61 (1993) 605, https://doi.org/ $10.1119 / 1.17221$

16. J. M. Lévy-Leblond, Electron Capture by Polar Molecules, Phys. Rev. 153 (1967) 1, https://doi.org/10.1103/ PhysRev.153.1

17. M. Ávila-Aoki, C. Cisneros, H. N. Núñez-Yépez, A. L. SalasBrito, Phys. Lett. A 373 (2009) 418. https://doi.org/ 10.1016/j.physleta.2008.11.054

18. C. Cisneros, R. P. Martínez-y-Romero, H. N. Núñez-Yépez, A. L. Salas-Brito, Limitations on the superposition principle: superselection rules in non-relativistic quantum mechanics, Eur. J. Phys. 19 (1998) 237, https://doi.org/10.1088/ 0143-0807/19/3/005. 